\title{
Rank und schlank: Schlangengurke
}

JENNIFER MARKWIRTH

\begin{abstract}
The snake gourd (Trichosanthes cucumerina) is a vining cucurbit native to India. The article describes its biological and morphological features and the use of its fruits. The plant is grown and displayed in the Tropicarium. Hints for its cultivation are given.
\end{abstract}

\section{Zusammenfassung}

Die Schlangenhaargurke (Trichosanthes cucumerina) ist ein rankendes Kürbisgewächs aus Indien. Biologie, Morphologie und Verwendung der Früchte werden vorgestellt. Die Art ist auch im Tropicarium zu sehen. Es werden kurze Hinweise zur Kultur gegeben.

\section{Lianen im Tropicarium}

Beim Betreten des Küsten- und Mangrovewälder-Hauses im Tropicarium tauchen unsere Besucherinnen und Besucher unmittelbar in eine tropische Welt ein. Die Temperaturen liegen hier deutlich über $20^{\circ} \mathrm{C}$, es ist schwülwarm, mehrere Wasserbecken sind vorhanden und überall gedeihen großblättrige tropische Gewächse. Im Sommerhalbjahr ranken verschiedene Lianen quer durch das Glashaus. Zum Winterhalbjahr hin werden sie zurückgeschnitten, sodass sie ab dem Frühjahr, wenn wieder bessere Lichtverhältnisse herrschen, erneut austreiben. Auffällig sind verschiedene kletternde Aristolochia-Arten (z. B. Aristolochia grandiflora, A. brasiliensis) mit ihren riesigen violettroten Blüten, die von Aasfliegen bestäubt werden.

Vor allem wenn sie fruchtet, lenkt zwischen den Aristolochien auch die Schlangengurke oder auch Schlangenhaargurke (Trichosanthes cucumerina) die Aufmerksamkeit auf sich. Die Schlangengurke ist ein Vertreter der asiatischen Gattung Trichosanthes, einem Kürbisgewächs.

\section{Eine Liane mit attraktiven, weiß bewimperten Blüten}

Die Urform der Schlangengurke stammt vermutlich aus Indien, wo sie zuerst in Kultur genommen und weiter gezüchtet wurde. Heute wird die einjährige Pflanze in vielen tropischen Ländern angebaut, wobei es sich um die Varietät anguina handelt, die gelegentlich auch als eigene Art $T$. anguina betrachtet wird. Die
Liane ist wärme- und feuchtigkeitsliebend, weshalb sie oft zu Beginn der Regenzeit ausgesät wird. Ihre Blätter sind handförmig gelappt. Die Ranken sind verzweigt und wie das Kabel an einem Telefonhörer spiralisiert, ähnlich wie die Ranken der Zaunrübe (Bryonia dioica).

Trichosanthes cucumerina ist einhäusig, d.h. es gibt männliche und weibliche Blüten auf einer Pflanze. Die männlichen stehen in Trauben, die weiblichen einzeln. Die Blüten sind weiß, haben eine lange Kronröhre, sind erst richtig in der Nacht geöffnet und duften in der Dunkelheit. Die Blütenblätter sind am Rand auffällig lang bewimpert. Darauf bezieht sich auch der Gattungsname, der sich von gr. trichos = Haar und anthos = Blüte ableitet. Trichosanthes-Blüten werden von Nachtfaltern bestäubt (Lieberei \& ReisdorfF 2012)

\section{Früchte}

Aus den Blüten gehen bis über $1 \mathrm{~m}$ lange Früchte hervor, die schlangenartig gebogen

Abb. 1 (oben links): Blüte bei Tag mit eingerollten Blütenblättern.

Abb. 2 (oben rechts): Seitenansicht der Blüte mit langer, schmaler Kronröhre.

Abb. 3 (unten links): Seitenansicht einer bei Nacht voll entfalteten Blüte.

Abb. 4 (unten rechts): Die gefransten Blüten sind bei Nacht voll geöffnet. 

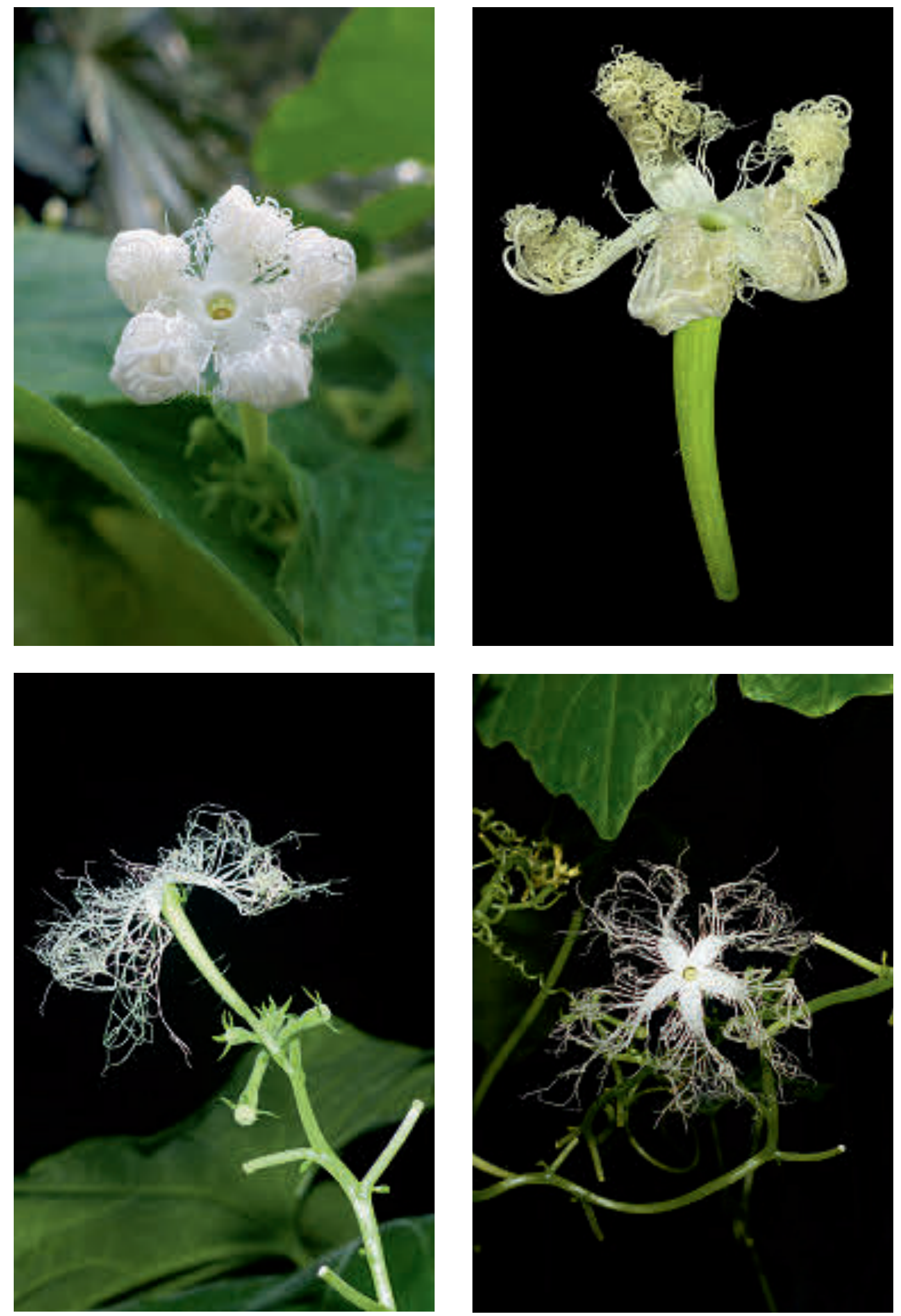

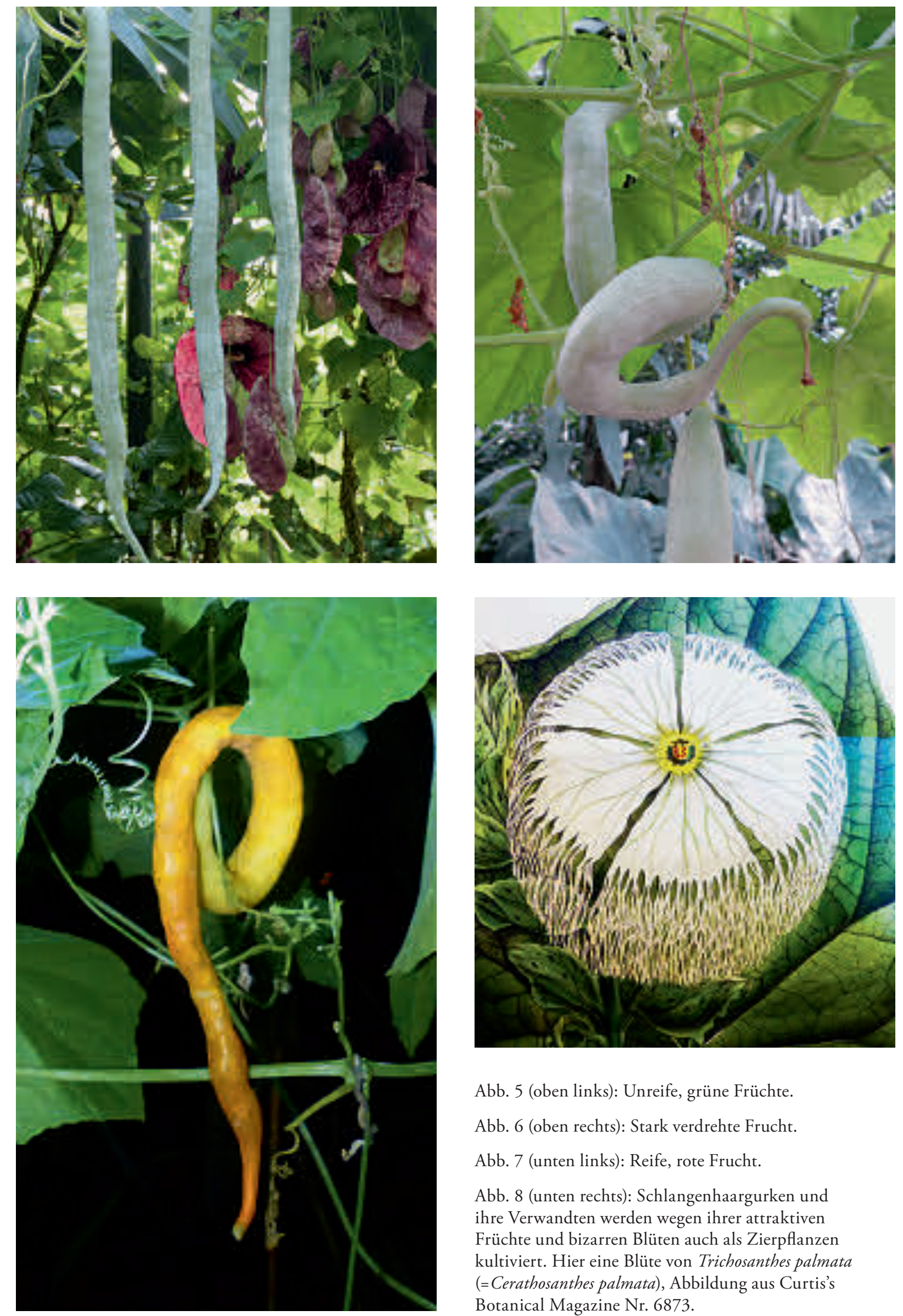

Abb. 5 (oben links): Unreife, grüne Früchte.

Abb. 6 (oben rechts): Stark verdrehte Frucht.

Abb. 7 (unten links): Reife, rote Frucht.

Abb. 8 (unten rechts): Schlangenhaargurken und ihre Verwandten werden wegen ihrer attraktiven Früchte und bizarren Blüten auch als Zierpflanzen kultiviert. Hier eine Blüte von Trichosanthes palmata (=Cerathosanthes palmata), Abbildung aus Curtis's Botanical Magazine Nr. 6873. 
und deutlich länger als bei der Wildform sind. Wenn sie als Gemüse kultiviert werden, hängt man ihnen gelegentlich kleine Gewichte (Steine) an das Fruchtende, um die Krümmung zu vermeiden und die Früchte noch länger werden zu lassen. Im Extremfall werden sie $2 \mathrm{~m}$ lang. Unreif sind sie grün mit weißen Sprenkeln, später orange bis gelb gefärbt. Das Fruchtfleisch ist etwas faserig. In ihm sind die dunklen Samen eingebettet. Da reife Früchte bitter schmecken, werden nur die etwa $30 \mathrm{~cm}$ langen unreifen grünen Früchte verarbeitet. Sie lassen sich ähnlich wie Zucchinis oder Auberginen zubereiten oder aber auch zu Chutneys verarbeiten. Spross- spitzen und Blätter liefern ein vitaminreiches Gemüse.

\section{Medizinische Verwendung}

Volksmedizinisch gilt die Pflanze in Ländern Süd- und Südostasiens wie Indien, Sri Lanka, Malaysia und Philippinen fast als Allheilmittel und kann gegen Würmer, aber auch bei Bronchitis, Hauterkrankungen, Durchfall, Fieber, Magenbeschwerden, Lebererkrankungen und andererseits sogar als Brechmittel oder Abtreibungsmittel eingesetzt werden. Wissenschaftlich nachgewiesen sind von diesen Anwendungen allerdings nur wenige und das auch nur in

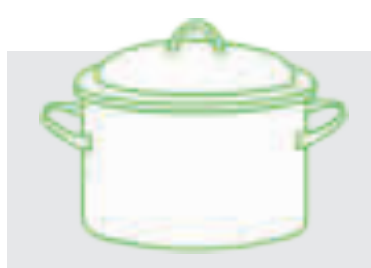

Zutaten für die Kokospaste

$1 / 2$ Kokosnuss grob gehackt

2 gehackte Knoblauchzehen

$1 / 2$ Teelöffel Kurkumapulver

nach Bedarf etwas Wasser zum

Verdünnen der Paste

Zutaten miteinander vermixen und mit einem

Schluck Wasser zu einer Paste vermengen.

\section{Zutaten für die Senfkornwürze}

1/2 Esslöffel Senfkörner

1 Strang Curryblätter

1 Esslöffel Pflanzenöl

Öl in einer kleinen Pfanne erhitzen und die Senfkörner darin platzen lassen. Die zerkleinerten Curryblätter hinzufügen.

\section{Zutaten für Erbsenmus (Tur Dal)}

2 Tassen gelbe Erbsen, über Nacht eingeweicht Erbsen mit Wasser kochen. Für eine Tasse Erbsenmus reicht eine Tasse Wasser.
Weitere Zutaten

1 kleine Schlangengurke, in Würfel geschnitten

$1 / 2$ Teelöffel Kurkumapulver

Salz nach Geschmack

1 Zwiebel

3 Knoblauchzehen, fein gehackt

$2 \mathrm{~cm}$ Ingwer, fein gehackt

1 Paprika, fein gehackt

1 Esslöffel Pflanzenöl

\section{Zubereitung}

Öl in einer mittleren Pfanne erhitzen und Zwiebel, Knoblauch, Ingwer, Paprika dazugeben und so lange anbraten, bis die Zwiebel weich ist. Salz und Kurkumapulver hinzufügen. Die Schlangenhaargurke hinzufügen und unter Rühren so lange erhitzen, bis sie weich ist. Erbsenmus hinzufügen und gut verrühren.

Die Kokospaste hinzugeben und eine Minute lang einrühren. Salz nach Geschmack dazugeben. Senfkornwürze hinzufügen und servieren.

verändert nach http://www.ayurananda.org/de/medialayurvedischKochen.pdf 

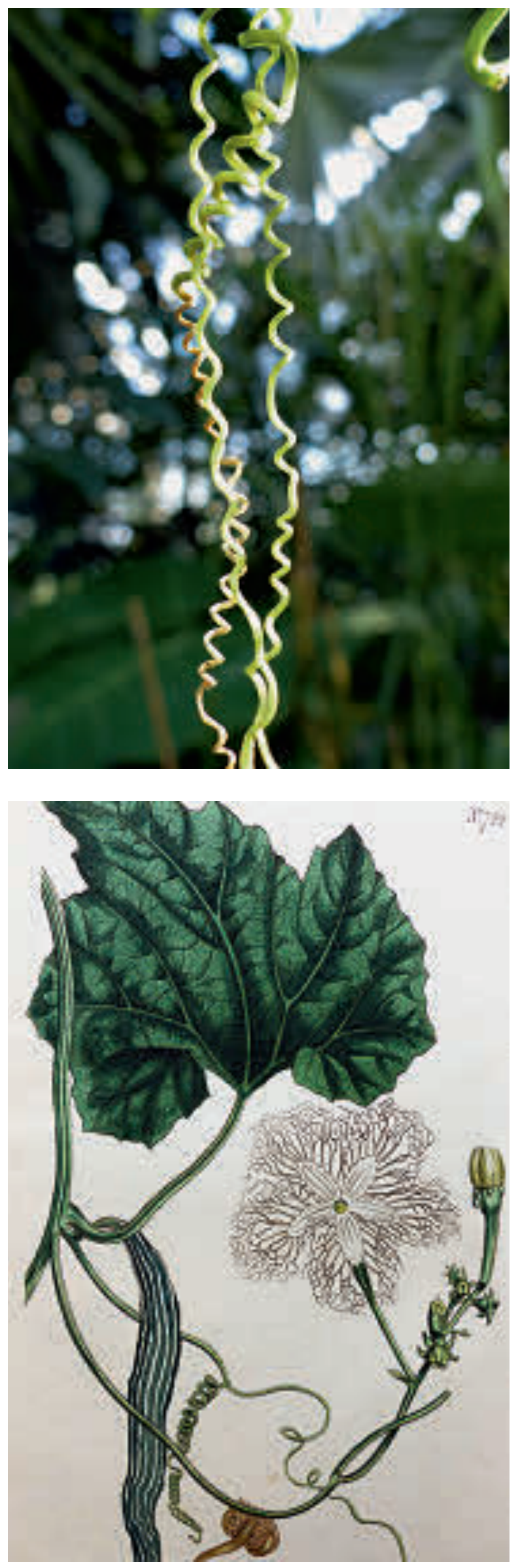

Laborversuchen, wobei Ergebnisse von Tierbzw. Zellkulturversuchen auch nicht immer auf den Menschen übertragen werden können. Untersuchungen haben gezeigt, dass Trichosanthes cucumerina durchaus als Entzündungshemmer oder als Schutz vor Leber- oder gastrointestinalen Schäden in Frage kommen kann. Ob Trichosanthes cucumerina allerdings tatsächlich auch gegen Brustkrebs hilft, muss erst noch genauer untersucht werden.

\section{Kultur der Schlangengurke in Haus und Garten}

Schlangenhaargurken lassen sich auch bei uns als einjährige Gemüsepflanzen kultivieren. Da sie es warm mögen und zum Keimen Temperaturen von mindestens $20^{\circ} \mathrm{C}$ benötigen, sollte man sie unbedingt im Haus vorziehen. Um die Keimung zu beschleunigen, wird das Einweichen der Samen für 1-2 Tage in lauwarmem Wasser oder das leichte Anfeilen der Samenschale empfohlen. Ausgepflanzt werden die Jungpflanzen nach den Eisheiligen an einen warmen, geschützten Ort. Die Pflanzen benötigen eine Rankhilfe. Die Pflanzen dürfen nie austrocknen, außerdem sollte regelmäßig gedüngt werden.

Trichosanthes dioica (Spitzgurke) ist eine enge Verwandte der Schlangenhaargurke. Die ausdauernde Art ist zweihäusig und wird in Indien ebenfalls als Gemüse sehr geschätzt. Sie ist gut verdaulich und soll den Kreislauf anregen.

\section{Dank}

Dank gilt IlsE ZüNDORF für Hinweise zur medizinischen Verwendung.

\section{Literatur}

Lieberei, R. \& ReisdorfF, C. 2012: Nutzpflanzen. 8. Aufl. - Stuttgart.

Abb. 9 (oben): Ranken an einer älteren Pflanze.

Abb. 10 (unten): Schlangenhaargurke, Abbildung aus Curtis's Botanical Magazine Nr. 722. 